\title{
EL PAPEL DE LA ACTIVIDAD FÍSICA Y EL DEPORTE COMO MEDIOS PARA EL DESARROLLO DE LA SALUD PSICOLÓGICA DE JÓVENES UNIVERSITARIOS.
}

Russell, L' ${ }^{1}$, Delgado, J ${ }^{2}$, Ortega, $A^{3}$

1. Russell González Leonel (2014). MsC en Psicología del Deporte Prof. Auxiliar y Consultante. Profesor Principal Disciplina Psicopedagogía Dpto. de Teoría y Metodología Universidad de Ciencias de la Cultura Física y el Deporte. M. Fajardo. Cuba. russell@inder.cu

2. Jorge Pensado Delgado (2014). Ph.D en Cultura Física y Deporte Prof. Auxiliar. Prof. de Psicología del Deporte. Disciplina Psicopedagogía. Dpto. de Teoría y Metodología Universidad de Ciencias de la Cultura Física y el Deporte. M. Fajardo. Cuba deportebienpensado@gmail.com

3. Arles Javier Ortega Parra (2014). Ph.D Ciencias de la Cultura Física. Docente programa Educación Física Recreación y Deportes. Universidad de Pamplona. arlesjavierortegap@hotmail.com

\section{RESUMEN}

Resulta de suma importancia el abordaje del papel que pueden ejercer las actividades de la Educación Física y la práctica del Deporte, en el caso que nos ocupa, el baloncesto, en la salud en general y en la salud psíquica o mental de los jóvenes universitarios que practican estos tipos de actividades de forma sistemática, en relación a aquellos que no las realizan. El poder determinar estas incidencias desde el punto de vista cuantitativo y cualitativo, permite a los profesores de educación física y del entrenamiento deportivo, realizar una mejor planificación de las cargas psicofísicas para sus deportistas y estudiantes y lograr incrementar los niveles de su preparación, aspectos estos que resultan significativos para el perfeccionamiento y mantenimiento de la salud. Es por ello que para la presente investigación se seleccionaron tres grupos de jóvenes universitarios: los que se dedicarían a la práctica del baloncesto, los que practicarían sistemáticamente la educación física y los que no realizarían ninguna de estas actividades. Los mismos fueron sometidos al estudio de un conjunto de indicadores psicomotrices mediante un diseño transversal caso control durante el cual los sujetos fueron medidos y evaluados, mediante las técnicas preceptúales, de las tensiones musculares, las reacciones simples, la reacción al objeto en movimiento y otras técnicas psicomotrices. Para el procesamiento de los resultados fue utilizado el modelo estadístico abreviado de 'URBAJ' que permitió obtener la media, la desviación, el coeficiente de variación, el error de la media, el grado de autenticidad de los resultados y comparar los grupos. Los resultados reflejaron que los sujetos sometidos a las variables independientes deporte baloncesto, el sexo y la práctica de la educación física, mejoraron cuantitativa y cualitativamente de forma notable en la mayoría de los parámetros de las variables psicomotrices, en comparación con los sujetos que no realizan estas actividades.

Palabras claves: salud mental, psicomotriz, actividades físicas, deporte

\section{THE ROLE OF PHYSICAL ACTIVITY AND SPORT AS MEANS FOR THE DEVELOPMENT OF PSYCHOLOGICAL HEALTH OF UNIVERSITY STUDENTS.}

\begin{abstract}
It is of a great importance addressing the role of physical education and sport practice in basketball playing, in general health and psychological or mental health for young university students who practice these types of activities in a systematically way in relation to those who do not perform them. The power to
\end{abstract}


determine these incidents in terms of quantity and quality ways, allows teachers of physical education and sports training to develop a better planning of psychophysical charges for their athletes and students and to increase levels of readiness. These aspects are significant for the development and maintenance of health. That is why for this research three groups of university students were selected: those devoted to basketball practice, those who practiced systematically physical education and those who would not do any of these activities. They were submitted to the study of a set of psychomotor indicators through a control case design during which individuals were measured and evaluated by perceptual techniques, muscle tension, simple reactions, reaction to moving object and other psychomotor skills. For the processing of the results was used the abbreviated statistical model 'URBAJ' that yielded the mean, deviation, coefficient of variation, the error of the mean, the degree of authenticity of the results and to compare groups. The results showed that students submitted to the independent variables, basketball sport, gender and practice of physical education, improved quantitatively and qualitatively in a markedly way in the most parameters of the psychomotor variables, compared with people who do not perform these activities.

Keywords: mental health, psychomotor, physical activity, sport

\section{INTRODUCCIÓN}

La salud constituye el aspecto más importante para mantener, elevar las potencialidades y alargar la vida de los seres humanos. Es más que conocida la definición que hizo la Organización Mundial de la Salud (1946) de la palabra "salud": "Un estado de completo bienestar físico, mental y social, y no solamente la ausencia de afecciones o enfermedades". Según esta definición, por tanto, el concepto de salud mental va implícito en el de salud en general y, además, como algo indisociable. De esta forma señala Knapp. E, (2007) que "el concepto de salud hay que entenderlo siempre en un sentido amplio e integral, pues es dinámico y está ligado a las condiciones de vida, a los avances científicos y a la evolución de la cultura. Así, las actitudes de la población respecto a la salud han ido cambiando conforme lo han hecho los valores sociales, de manera que hoy ya no sólo se tienen en cuenta los aspectos curativos de tiempos pasados, sino que se consideran otros como el entorno, los factores de riesgo, los estilos de vida, la educación sanitaria y todo cuanto contribuya a su fomento y promoción. Así pues, el concepto de salud debe entenderse también como la homeostasis entre el hombre y su medio ambiente, como una manera de vivir que suponga el desarrollo de la potencialidad humana, que permita el goce pleno y armonioso de sus facultades". Para Terris, M (1980) y L, Salleras (1985), "bienestar no siempre es salud; es una realidad difícil de alcanzar, es una definición estática que no toma en cuenta la salud como proceso, como algo dinámico y cambiante que se pude dar en grados muy diferentes en momentos distintos". San Martín. H, y Pastor (1984), al criticar la definición de la OMS señalan que "la salud no puede definirse hoy como un estado de bienestar absoluto, completo y estático, por cuanto representa una situación relativa, variable". Estos autores definen la salud como" un proceso social en su origen, que tiene repercusiones ecológicas en el ambiente de vida de la comunidad y que, finalmente produce en el individuo, según las circunstancias (favorables o negativas), el estado ecológico de salud o el desequilibrio ecológico que llamamos enfermedad'.

En cuanto a la salud psíquica o mental, no es fácil definirla. De hecho, según la OMS, no hay una definición oficial de la misma. Las diferencias culturales, las evaluaciones subjetivas y la competición de teorías profesionales hacen difícil definirla. Una aproximación a su conceptualización sería la siguiente: "La salud mental es un estado sujeto a fluctuaciones que obedecen a factores biológicos y sociales, en que el individuo se encuentra en condiciones de conseguir una síntesis satisfactoria de sus tendencias instintivas potencialmente antagónicas, así como de formar y mantener relaciones armoniosas con los demás y participar constructivamente en los cambios que pueden introducirse en su medio ambiente físico y 
social". En este sentido positivo, la salud mental es la base para el bienestar y el funcionamiento efectivo de la vida individual y de una comunidad, es mucho más que la ausencia de enfermedad mental. Este concepto, es coherente con su interpretación amplia y variada en las diferentes culturas. La salud psíquica o mental no es sólo la ausencia de trastornos mentales. Se define como un estado de bienestar en el cual el individuo es consciente de sus propias capacidades, puede afrontar las tensiones normales de la vida y puede trabajar de forma productiva y fructífera.

\section{Consideraciones sobre la actividad física, el deporte y su importancia en la salud psíquica o mental de los jóvenes}

La práctica de actividad física y deporte planificados pueden brindar una mejoría, y prósperidad a la salud y por tanto, contribuir a alargar la vida de los seres humanos en general y de los jóvenes en particular. La edad juvenil constituye la etapa del desarrollo psíquico donde el joven se manifiesta dentro de un conjunto de exigencias que condicionan la necesidad de lograr su inserción en la sociedad. La proyección hacia el futuro y la formación de la concepción del mundo se convierten en los principales resortes de desarrollo de dicha etapa. Domínguez, L (2000) "un aspecto fundamental de la concepción del mundo lo constituyen sus componentes morales, vistos como sistema de normas y valores que posibilitan la regulación interna del comportamiento en esa esfera moral, autónoma". Las prácticas de actividades físicas y deportivas ayudan a prevenir las enfermedades cardiovasculares y respiratorias; mejoran y aumentan la fuerza, la energía y pueden contribuir a reducir la ansiedad y la tensión psíquica. También resulta una buena manera de cambiar el rumbo de su estilo de vida. De esta forma el ejercicio físico y el bienestar mental están íntimamente relacionados. Se sabe, y múltiples investigaciones científicas lo han demostrado, que existe una relación directamente proporcional entre realizar actividad física y deporte de manera sistemática y el bienestar mental de las personas. En términos prácticos, quienes tomaron la decisión de cambiar el "control remoto por las zapatillas han tenido más energía, manejan mejor el estrés, tienen mayor concentración y motivación, se enojan y se frustran menos, duermen bien y tienen una vida social mucho más activa, es decir, la pasan mejor en todos los ámbitos, son más tranquilas y más plenas con sus vidas. La actividad física y el deporte siempre han estado considerados como medios importantes para estimular una gran cantidad de características de la personalidad de los jóvenes, de sus actitudes de gran valor individual y social, puntos de vistas, capacidades de acción y vivencias afectivas consideradas como deseables. Durante el proceso de la actividad físico-deportiva, además de la satisfacción de la necesidad de movimiento, surge una nueva necesidad, las tensiones físicas unidas a las tensiones psíquicas. En otras palabras, se reorganiza la necesidad misma de movimiento: esa es la necesidad no solo de moverse, sino también de experimentar con esta tensión la intensidad que mejor corresponda, la duración y el carácter de la actividad motriz. Martinó, C (1994) refiere que la "Educación Física no solo permite el desarrollo, la formación, la preparación y el perfeccionamiento físico, sino que además utiliza los deportes como uno de sus medios fundamentales para lograr la mayor potenciación de la integralidad funcional de los niños, adolescentes y jóvenes. Evidentemente este desarrollo no se produce en una sola dirección, sino que se manifiesta en todas las esferas psicofísicas y psicosociales que caracterizan la personalidad de los mismos". Esto puede ser un indicador del nivel de salud en general y de la salud psíquica o mental en particular. En sus investigaciones respecto a la dinámica de las funciones psíquicas en baloncestistas principiantes este autor encontró diferencias significativas entre niñas y niños que practicaban y no practicaban baloncesto.

La experiencia expuesta no ha sido estudiada en jóvenes universitarios en las mismas condiciones. Asimismo ha resultado insuficiente el tratamiento investigativo que se le ha dado a los estudios de las funciones psíquicas en los diferentes deportes en los cuales se han reducido los resultados a factores de rendimiento operativo en función de mejorar los componentes físicos, técnicos y tácticos, pero nunca la óptica ha sido hacia la consideración de que estos resultados reflejan niveles en la esfera de la salud psicomotriz de los sujetos involucrados en los estudios. Esta problemática se convierte en una necesidad que debe ser 
afrontada por los científicos y estudiosos de la Psicología del Deporte y otros, por cuanto ello se puede convertir a lo largo del tiempo de práctica no siempre bien planificada, en problemas de salud como los que han ocurrido con muchos atletas de alto rendimiento, que han padecido de diferentes afecciones no solo en la esfera biológica, sino también en la psicosocial, con repercusiones significativas en su personalidad. Son conocidos los problemas actuales del desentrenamiento como vía para enfrentar tales dificultades.

PROBLEMA CIENTÍFICO ¿En que grado influyen la actividad física y el deporte en el desarrollo de la esfera de la salud psicomotriz de los jóvenes universitarios practicantes?

\section{OBJETIVO GENERAL}

Evaluar las magnitudes de los posibles cambios producidos por la influencia de la actividad física y el deporte en el desarrollo de la esfera de la salud psicomotriz de los jóvenes practicantes.

\section{OBJETIVOS ESPECÍFICOS}

1-Determinar la magnitud de los posibles cambios en el desarrollo del campo visual de los jóvenes estudiados

2-Explorar las posibles fluctuaciones en el desarrollo de la percepción de profundidad en los jóvenes investigados

3-Determinar la medida de las posibles variaciones en el desarrollo de las reacciones en los jóvenes investigados

4-Valorar la magnitud de los posibles cambios en el desarrollo de las reacciones al objeto en movimiento en los jóvenes objeto de estudio

5 -Explorar las posibles fluctuaciones en el desarrollo de la percepción del movimiento en los jóvenes estudiados

6-Analizar las posibles variaciones en el desarrollo del esfuerzo percibido en los jóvenes investigados.

\section{METODOLOGÍA:}

\section{Organización de la investigación:}

La investigación se llevó a cabo en coordinación con el Departamento de Educación Física y
Deporte de La Universidad de La Habana, de la República de Cuba.

\section{Muestra.}

Para llevar a cabo la investigación fueron seleccionados de forma aleatoria 200 jóvenes entre hombres y mujeres de acuerdo a los objetivos propuestos. Esto dio comienzo a partir del curso escolar 2013-2014 y se propuso la conformación de 3 grupos de estudio, que practicarían sistemáticamente diferentes tipos de deportes y educación física tres veces por semana. De ellos, uno entrenaría baloncesto, otro las actividades de la Educación Física y el tercero, no practicaría ni deporte ni actividades físicas, dirigidas en los horarios de clases 0 de entrenamientos establecidos, producto de prescripción facultativa y por su no preferencia por estas actividades. Todo este proceso se realizó mediante el diagnóstico inicial de partida efectuado a dichos sujetos del nuevo ingreso al centro de estudio ya descrito. Para ello fueron utilizas las técnicas siguientes: la entrevista, análisis de documentos, la antropometría y los test psicológicos. En estas últimas técnicas fueron utilizados los resultados brindados por los especialistas, a partir de los estudios establecidos en el Departamento de Educación Física del centro ya referido implicado en la investigación.

Este profesional fue capacitado para enfrentar la Educación Física y el Entrenamiento en Baloncesto y además fue instruido con las orientaciones metodológicas esenciales de cómo dirigir los dos procesos tres veces por semana con el grupo A y B respectivamente. De esta forma se controló la variable profesor

\section{Conformación de los grupos}

De esta muestra de 125 sujetos que representó el $62,5 \%$ se seleccionaron también de forma aleatoria 20 en cada categoría es decir, 60 sujetos en general que representa el $48 \%$. De esta forma los grupos quedaron igualados en número, preferencias, edad, normalidad psicológica, antropométrica, el comienzo de los estudios universitarios y por el profesional que estaría trabajando con ambos grupos.

De esa forma se pudo asignar a los sujeto para cada grupo.

Grupo----A, constituido por (20 jóvenes mujeres) sin experiencia en la Educación Física 
Universitaria y prefirieron como práctica solo actividades en esta asignatura.

Grupo-B, constituido por (20 jóvenes hombres) sin experiencia en el deporte y prefirieron como práctica solo el baloncesto.

Grupo---C, constituido por (20 jóvenes hombres) que no practicaban ninguna de las actividades anteriores por prescripción facultativa y además, no mostraron interés por realizar dichas actividades.

\section{Diseño Metodológico de la Investigación}

En el caso de la investigación que nos ocupa, trabajamos con un diseño no experimental de tipo transeccional o transversal, específicamente el diseño caso control, que según Estévez y colaboradores (2004) este "se presenta cuando se quiere comparar dos grupos en los cuales uno presenta la característica 0 fenómeno objeto de estudio y el otro no la posee, a fin de determinar la influencia que puede haber entre la variable independiente y la dependiente". En esta experiencia se quiere establecer comparaciones entre el desarrollo psicomotor de los jóvenes de 19 anos que practican actividades de la Educación Física y el Deporte de Baloncesto, con aquellos que no practican ninguna de estas actividades. Es por ello que partiendo de grupos semejantes permite analizar el papel que tienen la 0 las variables independientes no manipuladas por el investigador sobre las manifestaciones externas de la variable dependiente que se aprecian en los sujetos mediante métodos de investigación.

\section{Métodos y Técnicas:}

El método utilizado para la investigación fue el de la medición en condiciones de laboratorio consistente en la evaluación de cada una de las variables mediante sus indicadores correspondientes en cada uno de los grupos estudiados, lo cual permitió tener un conocimiento preciso del estado de dichas variables, luego de tres meses de práctica sistemática en que cada grupo recibió la actividad planificada, es decir, el grupo A (hembras), Educación Física durante tres sesiones semanales. El grupo $B$ (varones) recibió el deporte de baloncesto durante tres sesiones semanales como las hembras y el grupo $\mathrm{C}$ (varones) que no recibió ninguna de las actividades planificadas a los otros grupos. De esta forma se trabajó con tres variables independientes (el deporte baloncesto, la educación física y el sexo), dos grupos experimentales y uno de control.

Las técnicas fueron aplicadas en el laboratorio de la Sección de Psicología del Deporte de La Universidad de Ciencias de Cultura Física Manuel Fajardo, donde existían las condiciones óptimas en cuanto a: espacio, temperatura, humedad, iluminación y equipamiento de investigación. Se analizaron con antelación todas las técnicas que serían objeto de prueba, de manera que se aplicasen aquellas que guardaban una mayor relación con el tipo de actividad a desarrollar por parte de los sujetos de la experiencia, tanto desde el punto de vista de la estructura funcional de las acciones como de las exigencias psicológicas que demandan el Baloncesto y la Educación Física.

\section{Las técnicas aplicadas fueron las siguientes: Técnica del Campo Visual.}

Instrumento: Perímetro visual. Español

Objetivo: Determinar el mayor ángulo periférico en el cual el sujeto comienza a percibir visualmente un objeto, sin tener en cuenta el color.

Instrucciones: Se les explicó a los sujetos que debían sentarse cómodos y apoyar la barbilla en el soporte del instrumento en la forma adecuada, fijar su visión hacia el punto central en el aparato y luego brindar la información de la identificación del objeto según el área de aparición. Debía repetir la acción tres veces en cada modalidad (abajo, arriba, externo, interno), de forma uníocular y vinocular.

Utilidad: Permite evaluar la magnitud subjetiva angular en que el sujeto es capaz de identificar una información visual, sin la discriminación del tipo de señal

\section{Técnica Percepción de la Profundidad.}

Instrumento: Túnel de Profundidad de Gowert. T.K.K. Japonés

Objetivo: Determinar la precisión en el cálculo de la profundidad visual uníocular y binocular.

Instrucciones: Se les explicó a los sujetos que debían sentarse cómodos y observar a través de la ventanilla del aparato ubicado a tres metros de distancia, el movimiento de la varilla que se desplazaba hacia la otra que se encontraba fija y debía indicar en que momento las varillas se encontraban a igual distancia de la perpendicular 
visual del sujeto perceptor. Debían repetir tres veces la acción en acercamiento y alejamiento.

Utilidad: Permite evaluar la magnitud subjetiva lineal del cálculo de la distancia entre dos objetos, con uno de ellos en movimiento.

\section{Técnica de Percepción del Movimiento}

Instrumento: Kinematómetro de Zhukovski. Cubano

Objetivo: Determinar el nivel de precisión en la sensibilidad músculo-motora durante la extensión de cada uno sus brazos horizontalmente

Instrucciones: Se les explicó a los sujetos que debían reproducir el movimiento de cada uno de sus brazos en posición horizontal, de acuerdo a la marca (patrón) establecida. Debía repetir la acción tres veces con cada miembro.

Utilidad: Permite evaluar la magnitud subjetiva del error en la reproducción de los movimientos en las extremidades superiores

\section{Técnica de la Percepción del esfuerzo}

Instrumento: Dinamómetro de mano. Español

Objetivo: Determinar la exactitud del esfuerzo percibido durante la ejecución de esfuerzos manuales máximos y diferenciales medios en ambas manos.

Instrucciones: Se les explicó a los sujetos que debían realizar un esfuerzo máximo, seguidamente en relación a este esfuerzo, calcular un esfuerzo medio (patrón) y posteriormente repetir tres veces dicho esfuerzo patrón que él estableció.

Utilidad: Permite evaluar la precisión subjetiva del esfuerzo durante las tensiones musculares manuales.

\section{Técnica de la Reacción psicomotriz}

Instrumento: Reacciómetro de pies y manos (Múltiple) T.K.K Japonés

Objetivo: Determinar la rapidez de la respuesta del sujeto ante la aparición de un estímulo visual percibido de forma inmediata.

Instrucciones: Se les explicó a los sujetos que debían responder con su mano diestra retirando lo más rápido posible esta del interruptor, ante la presencia inmediata del estímulo visual que apareciera en la pantalla del equipo. Debía repetir 10 veces la acción
Utilidad: Permite evaluar la capacidad de respuesta rápida del sujeto cuando tiene que tomar una decisión ante un estímulo visual cualquiera del medio ambiente

Técnica de la exactitud de la Reacción al objeto en movimiento

Instrumento: Electrosegundómetro de aguja T.K.K Japonés

Objetivo: Determinar la magnitud del error al reaccionar ante un objeto que se movía de forma uniforme

Instrucciones: Se les explicaba a los sujetos que debían detener lo más preciso posible en un punto predeterminado, una aguja que se desplazaba por una esfera graduada desde cero hasta $60^{\circ}$, manipulando un interruptor que le permitía realizar la acción. Debía repetir tres veces la acción

Utilidad: Permite evaluar la capacidad del sujeto para responder de forma precisa ante los objetos, fenómenos 0 personas que se mueven rápidamente en su radio de acción de manera uniforme.

En todos los casos, los resultados de las pruebas fueron recogidos y procesados mediante el Modelo Estadístico Operativo de 'URBAJ' el cual permite obtener los valores medios del grupo, la desviación, el coeficiente de variación, el error de la media y la autenticidad de los datos, obtenidos (el criterio de autenticidad que se asumió fue de alfa 0,05 como máximo). Se compararon los resultados de los grupos de tal forma que existiesen dos variables independientes, la educación física (grupo A) y el Entrenamiento en Baloncesto (grupo B). El (Grupo C) fue designado como control.

\section{ANÁLISIS $Y$ DISCUSIÓN DE LOS RESULTADOS}

Tabla -1 (Grupo A)

\begin{tabular}{|l|l|l|l|l|l|l|l|}
\hline \multirow{2}{*}{ OJOS } & Dirección & & X & Ex & Cv & Alf. & $\begin{array}{l}\text { X+ } \\
-\mathrm{D}\end{array}$ \\
\hline \multirow{4}{*}{ Derecho } & Externo & 72 & 16 & 3,6 & 0,3 & 0,001 & $\begin{array}{l}56- \\
88\end{array}$ \\
\cline { 2 - 8 } & Interno & 56 & 12 & 2,7 & 0,4 & 0,001 & $\begin{array}{l}44- \\
68\end{array}$ \\
\cline { 2 - 8 } & Abajo & 50 & 10 & 2,5 & 0,3 & 0,001 & $\begin{array}{l}40- \\
60\end{array}$ \\
\cline { 2 - 8 } & Arriba & & & & & & $35-$ \\
\hline
\end{tabular}




\begin{tabular}{|l|l|l|l|l|l|l|l|}
\hline & & 49 & 14 & 2,7 & 2,7 & 0,001 & 63 \\
\hline \multirow{4}{*}{ Izquierdo } & Externo & & & & & & $58-$ \\
& & 71 & 13 & 3,8 & 0,3 & 0,001 & 84 \\
\cline { 2 - 8 } & Interno & 59 & 17 & 3,9 & 0,4 & 0,001 & $\begin{array}{l}42- \\
76\end{array}$ \\
\cline { 2 - 8 } & Abajo & 54 & 14 & 3,0 & 0,3 & 0,001 & $\begin{array}{l}40- \\
68\end{array}$ \\
\cline { 2 - 8 } & Arriba & 49 & 11 & 2,3 & 0,2 & 0,001 & $\begin{array}{l}38- \\
60\end{array}$ \\
\hline
\end{tabular}

\section{Campo Visual:}

El análisis de los resultados obtenidos por cada grupo permite caracterizar la normalidad para cada dirección en ambos ojos, así como la tendencia relativa a la asimetría en el campo visual. Vemos en el grupo $\mathbf{A}$ de las féminas que practican educación física sistemáticamente (Tabla-1) que la comparación de los valores medios en los parámetros en cada ojo, permite inferir dos factores intrínsecos del desarrollo de esta capacidad: Primero; la tendencia cualitativa del desarrollo armónico bilateral de todos los parámetros estudiados, es decir, que el desarrollo máximo alcanzado describe un orden único para ambos ojos (externo, interno, abajo, arriba). Obsérvese que la tendencia descrita presenta un típico contenido, las magnitudes angulares se desarrollan en pares complementarios opuestos, predominan en cada par las direcciones de mayor ejercitación en cada etapa del desarrollo (la externa y la de abajo).

Segundo; la tendencia cuantitativa, refleja una ligera asimetría del campo visual hacia el ojo izquierdo, el cual se caracteriza por magnitudes angulares predominantes, de acuerdo con el estudio de los intervalos de normalidad.

\section{Tabla -2 (Grupo B)}

\begin{tabular}{|l|l|l|l|l|l|l|l|}
\hline OJOS & Dirección & X & D & Ex & CV & Alf. & $\begin{array}{l}\text { X+ } \\
- \text { D }\end{array}$ \\
\hline \multirow{2}{*}{ Derecho } & Externo & 77 & 10 & 3,0 & 0,1 & 0,001 & $\begin{array}{l}60- \\
70\end{array}$ \\
\cline { 2 - 8 } & Interno & 66 & 8 & 2,2 & 0,1 & 0,001 & $\begin{array}{l}58- \\
76\end{array}$ \\
\cline { 2 - 8 } & Abajo & 62 & 13 & 3,7 & 0,2 & 0,001 & $\begin{array}{l}49- \\
75\end{array}$ \\
\cline { 2 - 8 } & Arriba & 61 & 14 & 4,0 & 0,3 & 0,001 & $\begin{array}{l}47- \\
75\end{array}$ \\
\hline \multirow{2}{*}{ Izquierdo } & Externo & 86 & 6 & 1,4 & 0,1 & 0,001 & $\begin{array}{l}80- \\
92\end{array}$ \\
\cline { 2 - 8 } & Interno & 70 & 10 & 2,4 & 0,1 & 0,001 & $60-$ \\
\hline
\end{tabular}

\begin{tabular}{|l|l|l|l|l|l|l|l|}
\hline & & & & & & & 80 \\
\hline & Abajo & 65 & 8 & 2,1 & 0,1 & 0,001 & $\begin{array}{l}57- \\
73\end{array}$ \\
\hline & Arriba & 64 & 11 & 3,3 & 0,2 & 0,001 & $\begin{array}{l}53- \\
75\end{array}$ \\
\hline
\end{tabular}

En el grupo B de los varones que practican sistemáticamente deporte, específicamente baloncesto, se pude apreciar en la (Tabla-2) como se manifiestan los valores del desarrollo del campo visual. Al igual que el otro análisis se manifiestan dos factores semejantes. Primero; el desarrollo cualitativamente armónico entre los parámetros de ambos ojos, es decir, se confirma el desarrollo que esta manifestación produce en pares complementarios antagónicos con un orden descendente en el desarrollo máximo alcanzado, así como las direcciones rectoras en el desarrollo de cada par. Segundo, se manifiesta por la asimetría del campo visual hacia el ojo izquierdo con una magnitud predominante, según los límites en los intervalos de normalidad ya referidos. Este resultado constituye un índice interesante para el desarrollo de esta función psíquica y su análisis futuro.

Tabla -3 (Grupo C)

\begin{tabular}{|l|l|l|l|l|l|l|l|}
\hline OJOS & Dirección & X & D & Ex & CV & Alf. & $\begin{array}{l}\text { X+ } \\
- \text { D }\end{array}$ \\
\hline \multirow{7}{*}{ Derecho } & Externo & 68 & 7 & 1,9 & 0,1 & 0,001 & $\begin{array}{l}61- \\
75\end{array}$ \\
\cline { 2 - 8 } & Interno & 52 & 4 & 0,9 & 0,6 & 0,001 & $\begin{array}{l}48- \\
56\end{array}$ \\
\cline { 2 - 8 } & Abajo & 51 & 4 & 1,4 & 0,1 & 0,001 & $\begin{array}{l}49- \\
57\end{array}$ \\
\cline { 2 - 8 } & Arriba & 50 & 3 & 2,9 & 0,2 & 0,001 & $\begin{array}{l}50- \\
53\end{array}$ \\
\hline \multirow{5}{*}{ Izquierdo } & Externo & 67 & 7 & 2,4 & 0,1 & 0,001 & $\begin{array}{l}60- \\
67\end{array}$ \\
\cline { 2 - 8 } & Interno & 56 & 5 & 2,4 & 0,1 & 0,001 & $\begin{array}{l}51- \\
61\end{array}$ \\
\cline { 2 - 8 } & Abajo & 52 & 10 & 3,4 & 0,2 & 0,001 & $\begin{array}{l}42- \\
62\end{array}$ \\
\hline
\end{tabular}

En el grupo $\mathbf{C}$ de varones que no practican sistemáticamente baloncesto ni Educación Física, (Tabla-3) la comparación de los 
resultados del campo visual de ambos ojos, permite valorar lo siguiente: El desarrollo cualitativo de esta función psíquica es semejante en dos direcciones y se alternan en los parámetros de arriba y abajo; este hecho resulta interesante cuando analizamos los valores medios obtenidos en ambos ojos, pero en parámetros opuestos; lo cual puede deberse a mecanismos de compensación funcional en la recepción de los haces luminosos, que pueden tener incidencia directamente en la estructuración de las señales recibidas en estas direcciones 0 al desarrollo desarmónico de estos parámetros. Por otra parte, el análisis cuantitativo del campo visual nos permite plantear que la magnitud general, en grados, de la visión periférica difiere en el parámetro interno solamente en pequeños grados, por lo cual se puede suponer que no hay tendencia asimétrica significativa. A nuestro juicio, una diferencia pequeña en un parámetro del campo visual, es considerable; sin embargo, en el parámetro interno se evalúa con menor rigor científico, por cuanto esa diferencia puede estar influida por la convexidad más o menos pronunciada de la nariz de los sujetos. La dinámica del desarrollo del campo visual ojo derecho de los varones que practican baloncesto (G-1), con respecto a los que no practican el deporte y las féminas que si realizan actividades físicas, resulta significativa. En el parámetro arriba, los practicantes de baloncesto superan a los que no lo hacen en un $24 \%$ y a las féminas que si practican actividad física sistemática en un $16 \%$, significativo al 0.001 . El primer caso se justifica por el hecho que la práctica de este deporte requiere un ajuste en el control visual de la trayectoria del balón en los tiros al aro, pases largos, así como la ubicación visual del tablero, etc. El segundo, parece ser consecuencia de las diferencias propias del sexo.

En el parámetro abajo, los practicantes del deporte superan a sus coetáneos que no lo hacen en el $25 \%$ y a las féminas que si practican en el $14 \%$, significativo al 0,001. En el primer caso, el desarrollo alcanzado en esta dirección concuerda con las exigencias de controlar, en situaciones de entrenamiento y juego, el balón, tanto al driblar como en otras situaciones similares, mientras que el otro se debe a la variación psicofuncional típica de cada sexo.
En el parámetro externo se encuentran los mayores resultados medios obtenidos (0.001). Los baloncestistas superaron a los que no practican en el $13 \%$ y a las féminas que practican Educación Física en el 12\%. Este comportamiento es lógico y esperado que las magnitudes de este parámetro sean las más altas, porque todo el trabajo periférico lateral durante los entrenamientos y los juegos se realiza fundamentalmente en esa dirección.

En el parámetro interno, los practicantes del deporte superaron a ambos grupos en el $22 \mathrm{y}$ $20 \%$ al 0.001 . El desarrollo de esta dirección, aunque se encuentra restringida por la interrupción de la nariz, se origina, esencialmente, como complemento importante de la recepción de señales de la periferia lateral externa del ojo opuesto.

En el caso del ojo izquierdo de los practicantes del deporte (G-2), con respecto al resto de los jóvenes estudiados, se hace más evidente. En el parámetro arriba, superan a sus compañeros que no practican en el $22 \%$ y a las jóvenes que practican Educación Física en el 35\%, significativo al 0,001 . En el parámetro abajo, los que practican deporte superan a sus compañeros que no lo hacen en el $25 \%$ y a las hembras en el $23 \%$. En el parámetro externo es donde se encuentran los mayores resultados medios $(0,001)$. Los practicantes sistemáticos de baloncesto superan tanto a sus compañeros que no practican como a las féminas que solo lo hacen en la Educación Física (23 y 18\%) respectivamente. En el parámetro interno los practicantes de deporte superan a los no practicantes en el $19 \%$ respectivamente $(0,001)$. La práctica del deporte y de actividad física en los tres meses iniciales, etapa de adaptación a las cargas bio-funcionales y a la actividad específica, ha influido notablemente sobre el transformador de energía visual de estos sujetos. Indiscutiblemente, el analizador visual adquiere en estos sujetos un mayor desarrollo por que se prioriza su actividad, es decir, se hace rectora. Ocurre, así precisamente, porque el sujeto tiene que observar todas las demostraciones que el profesor realiza, además, debe controlar visualmente los movimientos de él y de todos los desplazamientos, lo que implica un gran ejercicio para la estructura óculo-motora; por supuesto, en la perspectiva psico-fisiológica observamos que las informaciones visuales van adquiriendo un matiz cada vez más 
especializado. La imagen visual de los movimientos, la imagen de la actividad que él realiza va siendo más nítida. Esto pude repercutir en el desarrollo de la actividad docente del sujeto.

En general, se puede inferir que las diferencias observadas entre los que practican baloncesto y las que también lo hacen en la Educación Física son esencialmente, consecuencia del sexo y de lo específico que desarrolla el tipo de actividad, por ende también el desarrollo ontogenético alcanzado hasta ese momento. Por su parte las diferencias observadas entre los que practican baloncesto y educación física y los que no lo hacen, se debe esencialmente a la influencia que el entrenamiento sistemático y la actividad física en general ejercen sobre estas funciones psíquicas. Por tanto estos resultados permiten caracterizar el desarrollo comparativo de la siguiente forma:

a-El entrenamiento sistemático influye en las diferencias de la función psíquica estudiada del 12 al $25 \%$, con un nivel de autenticidad de $95 \%$ o más al analizar los intervalos de normalidad.

b-El sexo y la práctica de actividades físicas influyen en las diferencias de la función psíquica analizadas del 13 y $35 \%$ con un nivel de autenticidad del $95 \%$ o más al analizar los intervalos de normalidad.

\section{Tabla-4}

\section{PARÁMETROS}

\begin{tabular}{|c|c|c|c|c|c|}
\hline & & Externo & Interno & Abajo & Arriba \\
\hline \multirow{3}{*}{$\begin{array}{c}\text { GRUPO } \\
\text { A }\end{array}$} & DERECHO & - & - & - & 0 \\
\hline & IZQUIERDO & $1^{\circ}$ & $4^{\circ}$ & & 0 \\
\hline & & & & $4^{\circ}$ & \\
\hline
\end{tabular}

\section{PARÁMETROS}

\begin{tabular}{|c|l|c|c|c|c|}
\cline { 3 - 6 } \multicolumn{2}{c|}{} & Externo & Interno & Abajo & Arriba \\
\hline \multirow{2}{*}{\begin{tabular}{c} 
GRUPO \\
\cline { 3 - 6 }
\end{tabular}} & DERECHO & - & - & - & - \\
\cline { 2 - 6 } & IZQUIERDO & $7^{0}$ & $4^{0}$ & $4^{0}$ & $4^{0}$ \\
\hline
\end{tabular}

\section{PARÁMETROS}

\begin{tabular}{|c|l|c|c|c|c|}
\cline { 3 - 6 } \multicolumn{2}{c|}{$\begin{array}{c}\text { GRUPO } \\
\text { C }\end{array}$} & Externo & Interno & Abajo & Arriba \\
\cline { 2 - 6 } & & $1^{\circ}$ & - & $2^{\circ}$ & - \\
\cline { 2 - 6 } & IZQUIERDO & - & $4^{\circ}$ & - & $2^{\circ}$ \\
\hline
\end{tabular}

En la Tabla-4 se ofrecen los resultados de las diferencias comparativas de cada parámetro, en ambos ojos, en los grupos estudiados.

En general el grupo A (féminas), presenta una inclinación muy leve hacia la asimetría, lo que demuestra la calidad de la información periférica bilateral como complemento de la integración de las imágenes en la percepción visual tendiente a lo normal, es decir al desarrollo armónico de todos los parámetros.

El grupo B presenta diferencias significativas en todos los parámetros. La dinámica de las diferencias adquiere, en esta ocasión, un matiz relativamente interesante. La anticipación de las señales de un ojo respecto al otro puede posibilitar ciertos mecanismos de asociación cortical que faciliten la estructura dinámica de la anticipación de las acciones en el juego de los baloncestistas, lo cual permitan, de seguir agudizándose el desarrollo asimétrico, establecer las relaciones específicas de esta función psíquica con la posición que el baloncestista ocupa y desempeña en el terreno. A nuestro juicio, la asimetría bidimensional de las imágenes percibidas se debe al proceso de adaptación del analizador visual a las condiciones específicas del medio en que se desenvuelve el deportista, a la influencia psicofisiológica del entrenamiento, 0 a la facilitación central de los procesos corticales de los centros correspondientes, durante el proceso de adaptación, a expensa de la reducción cuantitativa y cualitativa de otras funciones psíquicas

El grupo $\mathbf{C}$ por su parte presenta diferencias imperceptibles en los parámetros externos, abajo, arriba. Estos resultados permiten plantear que el desarrollo del campo visual en este grupo se manifiesta de manera normal.

\section{RESULTADOS DE LOS INDICADORES CORRESPONDIENTES AL NIVEL DE SALUD PERCEPTIVA EN PROFUNDIDAD EN LOS SUJETOS INVES TIGADOS}

Tabla-5 (Grupo A)

\begin{tabular}{|l|l|l|l|l|l|l|l|}
\hline \multirow{2}{*}{ OJOS } & $\begin{array}{l}\text { Direcció } \\
\mathrm{n}\end{array}$ & $\mathrm{X}$ & $\mathrm{D}$ & Ex & $\begin{array}{l}\mathrm{C} \\
\mathrm{v}\end{array}$ & Alf. & $\begin{array}{l}\mathrm{X}- \\
+\mathrm{D}\end{array}$ \\
\hline \multirow{2}{*}{ Derecho } & Acercam & $\begin{array}{l}6, \\
1\end{array}$ & $\begin{array}{l}3,5 \\
0\end{array}$ & $\begin{array}{l}0,7 \\
7\end{array}$ & 49 & $\begin{array}{l}000 \\
1\end{array}$ & $\begin{array}{l}2,6 \\
- \\
\end{array}$ \\
\cline { 2 - 8 } & Alejam. \\
\hline
\end{tabular}




\begin{tabular}{|c|c|c|c|c|c|c|c|}
\hline \multirow[t]{2}{*}{$\begin{array}{l}\text { Izquierd } \\
0\end{array}$} & $\begin{array}{l}\text { Acercam } \\
\text {. }\end{array}$ & $\begin{array}{l}6, \\
5\end{array}$ & $\begin{array}{l}2,8 \\
5\end{array}$ & $\begin{array}{l}0,6 \\
0\end{array}$ & 40 & $\begin{array}{l}000 \\
1\end{array}$ & $\begin{array}{l}3.7 \\
- \\
9,3\end{array}$ \\
\hline & Alejam. & $\begin{array}{l}4, \\
6\end{array}$ & $\begin{array}{l}2,6 \\
0\end{array}$ & $\begin{array}{l}0,5 \\
5\end{array}$ & 54 & $\begin{array}{l}000 \\
1\end{array}$ & $\begin{array}{l}2,0 \\
- \\
7,2\end{array}$ \\
\hline \multirow[t]{2}{*}{$\begin{array}{l}\text { Binocula } \\
r\end{array}$} & Acercam & $\begin{array}{l}3 \\
3\end{array}$ & $\begin{array}{l}2,3 \\
4\end{array}$ & $\begin{array}{l}0.5 \\
0\end{array}$ & 72 & $\begin{array}{l}000 \\
1\end{array}$ & $\begin{array}{l}0,1 \\
- \\
5,7\end{array}$ \\
\hline & Alejam. & $\begin{array}{l}2 \\
3\end{array}$ & $\begin{array}{l}1,0 \\
6\end{array}$ & $\begin{array}{l}0,5 \\
1\end{array}$ & 93 & $\begin{array}{l}000 \\
1\end{array}$ & $\begin{array}{l}1,2 \\
- \\
3,4\end{array}$ \\
\hline
\end{tabular}

Tabla-6 (Grupo B)

\begin{tabular}{|c|c|c|c|c|c|c|c|}
\hline & & & & & & & \\
\hline OJOS & Dirección & $x$ & $D$ & Ex & $\mathrm{Cv}$ & Alf. & $\begin{array}{r}X- \\
+D \\
\end{array}$ \\
\hline Derecho & Acercam. & 8,0 & 3,15 & 0,83 & 38 & 0,001 & $\begin{array}{l}4,9- \\
11\end{array}$ \\
\hline & Alejam. & 6,3 & 2,85 & 0,76 & 45 & 0,001 & $\begin{array}{l}3,4- \\
9,1\end{array}$ \\
\hline Izquierdo & Acercam. & 9,0 & 2,58 & 0,68 & 29 & 0,001 & $\begin{array}{l}6,4- \\
12\end{array}$ \\
\hline & Alejam. & 6,6 & 2,86 & 0,75 & 43 & 0,001 & $\begin{array}{l}3,7- \\
9,5\end{array}$ \\
\hline Binocular & Acercam. & 3,6 & 1,72 & 0,44 & 45 & 0,001 & $\begin{array}{l}1,9- \\
5,3\end{array}$ \\
\hline & Alejam. & 1,5 & 1,15 & 0,29 & 71 & 0,001 & $\begin{array}{l}0,4- \\
2,7\end{array}$ \\
\hline
\end{tabular}

Tabla-7 (Grupo C)

\begin{tabular}{|c|c|c|c|c|c|c|c|}
\hline OJOS & Dirección & $X$ & D & Ex & Cv & Alf. & $\begin{array}{l}X- \\
+D\end{array}$ \\
\hline \multirow[t]{2}{*}{ Derecho } & Acercam. & 9,4 & 4,42 & 1,30 & 47 & 0,001 & $\begin{array}{l}5,0- \\
14\end{array}$ \\
\hline & Alejam. & 7,5 & 4,75 & 1,47 & 65 & 0,001 & $\begin{array}{l}2,8- \\
12\end{array}$ \\
\hline \multirow[t]{2}{*}{ Izquierdo } & Acercam. & 10 & 3,79 & 1,20 & 39 & 0,001 & $\begin{array}{l}6,2- \\
14\end{array}$ \\
\hline & Alejam. & 9,8 & 7,59 & 2,40 & 79 & 0,001 & $\begin{array}{l}2,2- \\
17\end{array}$ \\
\hline \multirow[t]{2}{*}{ Binocular } & Acercam. & 4.0 & 1,60 & 0,51 & 42 & 0,001 & $\begin{array}{l}2,4- \\
5,6\end{array}$ \\
\hline & Alejam. & 4,5 & 3,79 & 1,20 & 89 & 0.001 & $\begin{array}{l}0,7- \\
8,3\end{array}$ \\
\hline
\end{tabular}

Alejam= alejamiento Acercam $=$ acercamiento

El análisis de los resultados del grupo $\mathbf{A}$, (Tabla-5) permite caracterizar esta función de la forma siguiente: los resultados del alejamiento del objeto son sensiblemente mejores en las tres modalidades. Esto indica que la acomodación del cristalino es mejor cuando los objetos de alejan o cuando los sujetos se separan de estos que son motivos de cálculo. Este mecanismo es inverso al que en la práctica deportiva se necesita ejecutar. Pensamos que lo expuesto caracteriza la posibilidad adaptativa del transformador visual a las exigencias de la actividad que se realiza. Los resultados obtenidos en la percepción binocular son significativamente mejores que los obtenidos en las modalidades unioculares. Cada parámetro de la percepción binocular es aproximadamente dos veces mejor que los mismos parámetros en cada ojo por separado. Por su parte en el grupo B, (Tabla-6), la caracterización es la siguiente: los resultados durante el alejamiento en relación con el acercamiento de los objetos son notablemente mejores. En comparación con el grupo A de las practicantes de Educación Física. Los baloncestistas presentan índices considerablemente aumentados. En el caso del grupo C, (Tabla-7), de los sujetos que no practican actividad física sistemática, los resultados reflejan que durante el alejamiento de los objetos, es mejor su comportamiento en las modalidades unioculares que en el acercamiento. Sin embargo en la modalidad binocular se presenta una mayor agudeza perceptual durante el acercamiento de los objetos. Obsérvese que estos resultados son inversos a los obtenidos por los sujetos que practican actividades física y deporte. No obstante se cumple que las modalidades binoculares son mejores que la uni oculares. En sentido general con esto se justifica el hecho de que la interacción de las imágenes de cada ojo a nivel cortical permite el cálculo de las distancias absolutas en la profundidad con mayor precisión. El análisis de los coeficientes de variación nos permite inferir que el desarrollo de la percepción de profundidad se puede aumentar considerablemente y que esta función está determinada esencialmente por el tipo de actividad que realice el sujeto. Los índices de normalidad, en general, están en correspondencia con la dinámica de los resultados medios analizados con anterioridad.

(G-3, 4 y 5 ).

\section{RESULTADOS DE LOS INDICADORES DE LA SENSIBILIDAD MÚCULO-MOTORA CORRESPONDIENTE AL NIVEL DE SALUD EN LA PERCEPCIÓN DE LOS MOVIMIENTOS DE LOS SUJETOS INVESTIGADOS}

Tabla -8

\begin{tabular}{|c|c|c|c|c|c|c|c|}
\hline \multirow{2}{*}{ BRAZOS } & GRUPOS & X & D & Ex & Cv & Alf. & $\begin{array}{c}\text { X+ } \\
\text { - D }\end{array}$ \\
\hline \multirow{2}{*}{ Derecho } & $\mathrm{A}$ & 5,5 & 2,87 & 0,62 & 52 & 0001 & $\begin{array}{l}2,6- \\
8,4\end{array}$ \\
\cline { 2 - 8 } & $\mathrm{B}$ & 5,0 & 2,59 & 0,69 & 51 & 0001 & $\begin{array}{l}2,4- \\
7,6\end{array}$ \\
\hline
\end{tabular}




\begin{tabular}{|c|c|c|c|c|c|c|c|}
\hline & $C$ & 9,8 & 2,83 & 0,89 & 28 & 0001 & $\begin{array}{l}7,0- \\
13\end{array}$ \\
\hline \multirow[t]{3}{*}{ Izquierdo } & A & 5,0 & 2,09 & 0,45 & 41 & 0001 & $\begin{array}{l}2,9- \\
7,0\end{array}$ \\
\hline & $B$ & 4,6 & 2,88 & 0,77 & 62 & 0001 & $\begin{array}{l}1,7- \\
7,5\end{array}$ \\
\hline & $C$ & 7,1 & 4,73 & 1,49 & 65 & 0001 & $\begin{array}{l}2,4- \\
12\end{array}$ \\
\hline
\end{tabular}

En esta modalidad se presentan variaciones en los resultados obtenidos en ambas extremidades superiores (derecha e izquierda) en las hembras y varones que practican actividades físicas y deporte. Las diferencias son más evidentes en las hembras del grupo $\mathbf{A}$. En la (Tabla-8) se pueden observar los resultados. Al comparar los valores medios en cada brazo nos percatamos de una ligera tendencia hacia la reducción de los errores en el brazo izquierdo. Es interesante esto porque toda las jóvenes poseen una mayor efectividad en el brazo derecho, lo que nos hace pensar que la influencia de la actividad repercute en la simetría motriz de los miembros superiores. Sin embargo las posibilidades de desarrollo son más elevadas en el brazo derecho, por lo que podemos asumir que el desarrollo psicomotor evoluciona en esta etapa hacia la asimetría bilateral en los miembros superiores. En efecto, los varones del grupo $\mathbf{B}$ y las hembras del $\mathbf{A}$, presentan índices de desarrollo en la percepción del movimiento que distan mucho de poderse evaluar como buenos en cuanto a la exactitud, pero evidentemente resultan mejores en comparación con el grupo $\mathbf{C}$ en sentido general. Las acciones durantes los entrenamientos y las prácticas de actividades de la educación física, exigen el aumento de la precisión en los movimientos con las extremidades superiores, es decir, el control vasomotor y la participación de la información kinestésica permiten la adecuación psicomotora de los sujetos a este tipo de actividades, estableciéndose de esta forma adaptaciones al micro-ambiente, lo cual facilita la precisión en la percepción de los movimientos propios ejecutados por ello. (G-6)

\section{RESULTADOS DE LOS INDICADORES DEL ESFUERZO CORRESPONDIEN TES AL NIVEL DE SALUD EN LA PERCEPCIÓN DEL ESFUERZO DE LOS SUJETOS INVESTIGADOS}

Tabla -9

\begin{tabular}{|l|l|l|l|l|l|l|l|}
\hline MANOS & GRUPO & & & & & & $\mathrm{X}_{+}$ \\
\hline
\end{tabular}

\begin{tabular}{|c|c|c|c|c|c|c|c|}
\hline & $\mathrm{S}$ & $X$ & D & Ex & $\begin{array}{l}\text { C } \\
\text { V }\end{array}$ & Alf. & $-D$ \\
\hline \multirow[t]{3}{*}{$\begin{array}{l}\text { Derecha } \\
\text { Máximo }\end{array}$} & $A$ & $\begin{array}{l}18, \\
5\end{array}$ & $\begin{array}{l}3, \\
0\end{array}$ & $\begin{array}{l}0,6 \\
2\end{array}$ & 21 & $\begin{array}{l}000 \\
1\end{array}$ & $\begin{array}{l}16- \\
21\end{array}$ \\
\hline & $B$ & $\begin{array}{l}19, \\
3\end{array}$ & $\begin{array}{l}4, \\
3\end{array}$ & $\begin{array}{l}1,1 \\
4\end{array}$ & 22 & $\begin{array}{l}000 \\
1\end{array}$ & $\begin{array}{l}15- \\
24\end{array}$ \\
\hline & C & $\begin{array}{l}21, \\
3\end{array}$ & $\begin{array}{l}4, \\
1\end{array}$ & $\begin{array}{l}1,2 \\
9\end{array}$ & 19 & $\begin{array}{l}000 \\
1\end{array}$ & $\begin{array}{l}17- \\
25\end{array}$ \\
\hline \multirow{3}{*}{$\begin{array}{l}\text { Derecha } \\
\text { Diferenc } \\
\text {. }\end{array}$} & $A$ & 1,9 & $\begin{array}{l}1, \\
3\end{array}$ & $\begin{array}{l}0,2 \\
8 \\
\end{array}$ & 68 & $\begin{array}{l}000 \\
1 \\
\end{array}$ & $\begin{array}{l}0.6 \\
- \\
3,2 \\
\end{array}$ \\
\hline & $B$ & 2,2 & $\begin{array}{l}1, \\
1\end{array}$ & $\begin{array}{l}0,2 \\
9\end{array}$ & 50 & $\begin{array}{l}000 \\
1\end{array}$ & $\begin{array}{l}1,1 \\
- \\
3,3 \\
\end{array}$ \\
\hline & $C$ & 2,0 & $\begin{array}{l}1, \\
8\end{array}$ & $\begin{array}{l}0,5 \\
5\end{array}$ & 90 & $\begin{array}{l}000 \\
1\end{array}$ & $\begin{array}{l}0,2 \\
- \\
3,8\end{array}$ \\
\hline
\end{tabular}

\begin{tabular}{|c|c|c|c|c|c|c|l|}
\hline \multirow{3}{*}{$\begin{array}{l}\text { Izquierda } \\
\text { Máximo }\end{array}$} & $\mathrm{A}$ & 18,1 & 3,4 & 0,74 & 18 & 0001 & $\begin{array}{l}15- \\
22\end{array}$ \\
\cline { 2 - 8 } & $\mathrm{B}$ & 18,5 & 3,1 & 0,82 & 16 & 0001 & $\begin{array}{l}15,- \\
22\end{array}$ \\
\cline { 2 - 8 } & $\mathrm{C}$ & 21,4 & 3,1 & 0,98 & 14 & 0001 & $\begin{array}{l}18- \\
25\end{array}$ \\
\hline \multirow{2}{*}{$\begin{array}{l}\text { Izquierda } \\
\text { Diferenc. }\end{array}$} & $\mathrm{A}$ & 1,7 & 0,7 & 0,15 & 41 & 0001 & $\begin{array}{l}1,0- \\
2,4\end{array}$ \\
\cline { 2 - 8 } & $\mathrm{B}$ & 1,7 & 0,8 & 0,21 & 47 & 0001 & $\begin{array}{l}0,9- \\
2,5\end{array}$ \\
\cline { 2 - 8 } & $\mathrm{C}$ & 1,7 & 0,9 & 0,28 & 53 & 0001 & $\begin{array}{l}0,8- \\
2,6\end{array}$ \\
\hline
\end{tabular}

En el grupo $\mathbf{A}$ de las hembras que practican Educación Física sistemática (Tabla-9), se observan ligeras diferencias en los índices de fuerza máxima y del esfuerzo diferencial al comparar los resultados de la mano derecha con los de la izquierda, no significativos, ocurriendo lo mismo en la dosificación de la fuerza media. Estos resultados reflejan el desarrollo bilateral armónico en esta modalidad. En el grupo $\mathbf{B}$ de varones que practican baloncesto, se observan ligeras diferencias en los índices medios de la fuerza máxima y esfuerzo diferencial al comparar los resultados de la mano derecha con los de la precisión en la reproducción de los esfuerzos en la mano izquierda. Al parecer, el desarrollo de la fuerza en esta etapa, son semejantes para ambos miembros. En el grupo de los sujetos que no practican actividades físicas ni deporte, grupo $\mathbf{C}$, las diferencias en los resultados medios de la fuerza máxima y esfuerzo diferencial resultan imperceptibles.

$\begin{array}{lcccr}\text { RESULTADOS } & \text { DEL } & \text { NIVEL } & \text { DE } & \text { LA } \\ \text { DOSIFICACIÓN } & \text { DE } & \text { LAS } & \text { TENSIONES } \\ \text { MUSCULARES } & \text { DE } & \text { LOS } & \text { SUJETOS } \\ \text { ESTUDIADOS } & & & \end{array}$


TABLA-10

\begin{tabular}{|c|c|c|c|c|}
\hline GRUPOS & \multicolumn{2}{|c|}{$\begin{array}{c}\text { ERRORES DEL } \\
\text { CÁLCULO }\end{array}$} & \multicolumn{2}{c|}{$\begin{array}{c}\text { ERRORES DE } \\
\text { REPRODUCCIÓN }\end{array}$} \\
\hline A & 2,7 & 1,9 & 0,2 & 0,2 \\
\hline B & 2,2 & 2,3 & 1,6 & 0,8 \\
\hline C & 4,7 & 5,0 & 0,5 & 0,2 \\
\hline MANOS & DERECHA & IZQUIERDA & DERECHA & IZQUIERDA \\
\hline
\end{tabular}

Como puede apreciarse en la (Tabla-10), la capacidad de calcular los esfuerzos es mejor en los baloncestistas que en los que no practican este deporte; sin embargo, se manifiesta el proceso inverso cuando analizamos la capacidad de dosificar los esfuerzos al reproducir la tensión media calculada (G-7). Los resultados de los grupos A y $\mathbf{B}$ son semejantes y se presenta muy alejado dicho resultado en el grupo $\mathbf{C}$, es decir, el cálculo de estos esfuerzos es mejor en los primeros grupos., a pesar de que presenta una característica más estable en las reproducciones aun cuando estas se presentan influidas notablemente por una deficiencia muy marcada en la estimación del esfuerzo medio calculado. (E, M, C).

Los resultados que muestra esta tabla correspondiente a la capacidad de los sujetos para responder a estímulos visuales externos de forma rápida, nos permiten plantear que los grupos estudiados no presentan diferencias significativas desde el punto de vista estadístico en esta variable, pero si los varones baloncestistas presentan mejor resultado que las hembras y los no practicantes de estas actividades. Al parecer la reacción simple no resulta una función psico-fisiológica indicativa de diferencias entre los sujetos estudiados, a pesar del tipo de actividad que se practique, pues no produce cambios significativos con relación a los sujetos que no realizan actividades físicas sistemáticas, llámese deporte o educación física. Estos resultados coinciden con los hallados por Martinó. C y L. Russell (1994) estudiando la influencia de la Orientación de la Atención en las Reacciones Psicomotoras en motociclistas y luchadores de alto rendimiento.

No obstante sí es importante estudiar la estabilidad de las curvas en función de las repeticiones en el (G-8), donde se muestra la manifestación de dicha estabilidad de cada grupo, lo cual si representa un dato importante desde el punto de vista cualitativo. De esta forma la mejor estabilidad la alcanza el grupo B, en tanto son muy bruscas las variaciones en el grupo $\mathbf{A}$ y $\mathbf{C}$ respectivamente. La estabilidad si constituye un buen indicador para conocer sobre el nivel de influencia de la carga física sobre la esfera psíquica del sujeto, lo cual permite orientar mejor la distribución de las cargas en la preparación.

RESULTADOS DE LA CAPACIDAD DE REACCIÓN DE LOS SUJETOS ESTUDIADOS TABLA-11

\begin{tabular}{|c|c|c|c|c|c|c|}
\hline GRUPOS & $X$ & D & Ex & CV & Alf. & $\begin{array}{c}X- \\
+D\end{array}$ \\
\hline A & 0,28 & 0,03 & 0,006 & 2,3 & 0001 & $\begin{array}{l}0,25- \\
0,31\end{array}$ \\
\hline B & 0,26 & 0,04 & 0.009 & 14 & 0001 & $\begin{array}{l}0,22- \\
0,30\end{array}$ \\
\hline C & 0,27 & 0.02 & 0.005 & 8,1 & 0001 & $\begin{array}{l}0,25- \\
0,29\end{array}$ \\
\hline
\end{tabular}

\section{RESULTADOS DEL NIVEL DE EXACTITUD EN LA REACCIÓN}

TABLA-12

\begin{tabular}{|c|c|c|c|c|c|c|}
\hline GRUPOS & $X$ & D & Ex & Cv & Alf. & $\begin{array}{l}X- \\
+D\end{array}$ \\
\hline A & 0,31 & 0,08 & 0,007 & $2^{4}$ & 0,05 & $\begin{array}{l}0,23- \\
0,15\end{array}$ \\
\hline B & 0,20 & 0,06 & 0,001 & 7,9 & 0,001 & $\begin{array}{l}0,14- \\
0,26 \\
\end{array}$ \\
\hline C & 0,23 & 0,06 & 0,093 & 61,4 & 0,001 & $\begin{array}{l}0,17- \\
0,29\end{array}$ \\
\hline
\end{tabular}

Diferencias no Significativas $(+)$

Reacción al Objeto en Movimiento (Tabla -12) En esta tabla se exponen los datos finales de la exactitud de la reacción al objeto en movimiento; es evidente la similitud en los 
resultados medios inter-grupos y también similares al comportamiento de la reacción simple en (Tabla-11). A pesar de esto, el análisis de las curvas típicas nos revela la dinámica que estos valores presentan (G-9). Esta curva en el grupo B aun cuando el resultado es equivalente al de la norma planteada, los resultados reales obtenidos por los sujetos en cada repetición distan mucho de estar ajustados a la misma, es decir; los datos se comportan como reacciones de anticipación o retardada, lo que denota cierta inestabilidad y tipifica, de esta forma, la calidad deportiva de los sujetos de este grupo. En el caso del grupo A hay inestabilidad creciente, caracterizada por la incidencia de respuestas retardadas consecutivas de índole progresiva, que evidencia el aumento de los errores. Por su parte el grupo $\mathbf{C}$, tiene inestabilidades $\mathrm{y}$ estabilidades relativas, que pueden considerarse como ajustadas en cuanto a la compensación psicomotora durante la actuación.

Con anterioridad habíamos planteado la influencia que el deporte baloncesto ejercía sobre la estructura óculo-motora, lo cual supone se presente una cierta alteración en el mismo sentido en las reacciones simples y en la exactitud al objeto en movimiento, por cuanto ambas utilizan las informaciones visuales. De los resultados obtenidos podemos inferir que la práctica del baloncesto influye notablemente en la calidad de las imágenes percibidas por los sujetos, pero no ocurre así al analizar las manifestaciones del analizador visual en función de las reacciones. Si la influencia expresada por la interacción de los analizadores se ejerciera en forma más acentuada en el plano fisiológico, no cabe dudas que el tiempo de la reacción sería mejor en los practicantes de baloncesto; tales reacciones serían más exactas y se encontrarían muy distantes en cuanto al porcentaje significativo en relación a los sujetos que no se dedican a este tipo de actividades.

En la (Tabla-13) se presentan los resultados generales de la investigación. La influencia ejercida por las variables sexo y práctica de la Educación Física sobre las funciones psíquicas estudiadas, resulta definitivamente marcada en el área del campo visual y de la percepción de la profundidad; mientras que la influencia ejercida por la variable entrenamiento en baloncesto se generaliza a casi tos los parámetros investigados.

\section{CONCLUSIONES}

El baloncesto, como deporte, contribuye al desarrollo de la visión periférica y de la percepción de profundidad. Bajo la influencia del entrenamiento se aumenta el campo perceptivo visual en todos los parámetros y crece la exactitud de la percepción de profundidad durante el acercamiento y el alejamiento de los objetos.

El estudio del campo visual en los jóvenes que practican o no baloncesto o educación física ha demostrado que sus magnitudes se encuentran en dependencia directa del sexo y el tipo de actividad que realice el sujeto

a- El campo visual de los varones que practican baloncesto es mayor que el de las hembras que practican educación física (promedio del $15 \%$ ) b-El campo visual de los varones que practican baloncesto está aumentado en comparación con los que no lo practican (promedio $20 \%$ )

El desarrollo de la percepción de profundidad de los varones se manifiesta en el cambio desigual de la exactitud en el cálculo, dependiendo de que practiquen baloncesto o educación física.

a-La exactitud de la percepción visual de la profundidad binocular de los varones y hembras que practican sistemáticamente actividades físico-deportivas, resulta similar, mientras que la de los sujetos que no las practican, la magnitud del error es considerablemente mayor. (promedio $86 \%$ )

b-El rasgo característico de la medición de la profundidad de los varones que practican baloncesto consiste en la mayor exactitud de la percepción durante el alejamiento del objeto en movimiento que durante el acercamiento, mientras que la exactitud de la percepción de los que no practican este deporte, es peor durante el alejamiento de los objetos.

c-La exactitud de la medición visual de la profundidad es significativamente mayor en la visión binocular que en la visión uniocular. (promedio $135 \%$ )

La práctica sistemática del baloncesto estimula notablemente el desarrollo de la percepción del movimiento, así como que depende del sexo y de la práctica de las actividades físicas las 
cuales influyen moderadamente en sus diferencias

5-La capacidad de regular las tensiones musculares depende muy poco del sexo y el tipo de regulación depende de la actividad que el sujeto realice, esto se desarrolla acentuadamente como consecuencia de la práctica sistemática de las actividades físicodeportivas, en especial del baloncesto, pero puede verse interferida como consecuencia de la adaptación a la carga psicofisiológica del entrenamiento.

La velocidad de reacción simple no es un indicador que permita caracterizar la actividad de los baloncestistas ni de los practicantes de la educación física, por cuanto no sufre cambios significativos en función de las variables influyentes.

La exactitud de la reacción al objeto en movimiento no sufre cambios significativos atribuibles al sexo y a la práctica de actividades físicas y deporte, en la etapa estudiada.

Las funciones psícomotrices estudiadas se desarrollan en relación con el sexo en un 72,6 $\%$ y de la estructura de la actividad que se practique en un $77,1 \%$., con una tendencia significativa a la evolución compensada.

\section{REFERENCIAS BIBLIOGRAFICAS}

Canizares Hernández, Marta. (2008). La Psicología en la actividad física. Su aplicación en la educación física, el deporte, la recreación y la rehabilitación. Ciudad de La Haba na. Editorial Deportes.

Constitución de la Organización Mundial de la Salud (1946). Conferencia Internacional de Salud. http://apps.who.int/gb/bd/PDF/bd47/SP/c onstitucion-sp.pdf

Domínguez. Laura, (2000) Ponencia presentada en mesa redonda sobre los problemas de la periodización del desarrollo, XXI Congreso Interamericano de Psicología. La Habana Cuba. Palacio de las Convenciones

Estévez, C "y Cols" (2004) La Investigación Científica en la Actividad Física: su Metodología. La Habana. Cuba. Editorial Deportes.
Gorbunov. B. D, Guenadi, (1988) Psicopedagogía del Deporte. Moscú. Editorial Cultura Física y Deportes. URSS.

Iriarte. S 'y Cols' (2011). Actividad física y salud mental, ¿dúo inseparable? Metas de enfermería, Vol. 14, №. 5 , págs. $64-69$

Knapp, Rodríguez. Elisa (2007) Psicología de la Salud. Editorial Felix Varela. Ciudad de La Habana.

Martinó, C 'y Cols' (1994) Sobre Psicología del Deporte. Suplemento No.15. Boletín CientíficoTécnico. Inder. Cuba

Needle. R; J. F. Sallis; and C. B Taylor (1985). "The relation of physical activity and exercise to Mental health. Public Health Rep. p. 195-202

Puni, A. Z y T.T. Changarov (1979). Psicología de la Educación Física y el Deporte. Editorial Científico Técnica. Ciudad de La Habana

Terris. M (1980) y L, Salleras (1985) La revolución epidemiológica y la medicina social. Ed. Siglo XXI, Madrid.

San Martín. H, y Pastor (1984). Salud comunitaria. Teoría y Práctica, Díaz de Santos. Madrid. 